\title{
Le réalisme magique dans La jument verte de Marcel Aymé et Nawat Alkarm de Nagwa Chaban
}

\section{Par}

\section{Drl Ahmed Moawad Abd-Elhadi}

Maître de conférences en littérature française à la faculté des lettres Département de français Université d'El-Arich 


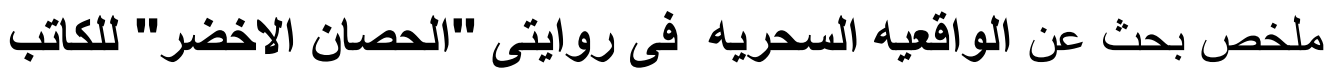
الفرنسى "مارسيل ايميه" ونوة الكرم للكاتبه المصريه لونيه نجوى شعبان

اتسم أدب كل من مارسيل ايميه و نجوى شعبان بالغر ابة و السحرية حيث

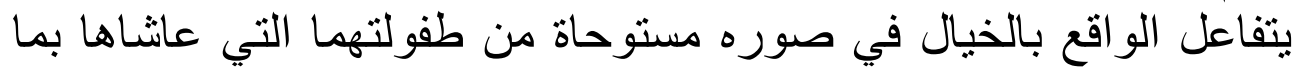

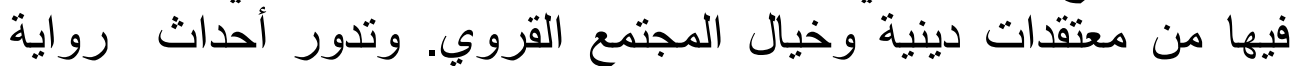

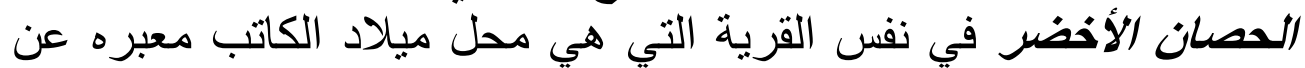

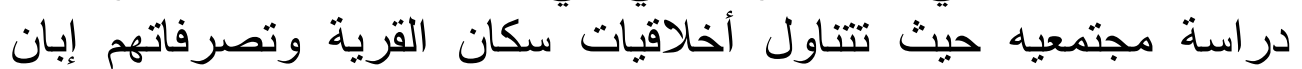
القرن التاسع عشر. فى حين ان روايه نوة الكرم تدور احداثها فى مدينه دمياط المصريه والتي هي ايضا محل ميلاد الكاتبه معبره عن دراست استه مجتمعيه تتناول عادات وسلولك سكان المدينة وتصرفاتهم إبان القرن الترن

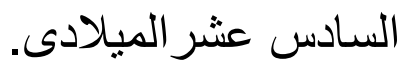

و الهُف من هذه الدراسة هو إلقاء الضوء على بعض بعض النظريات

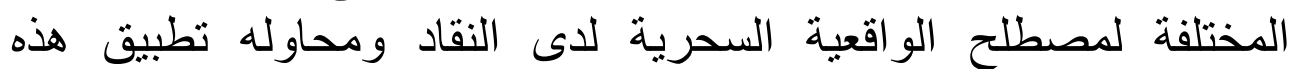

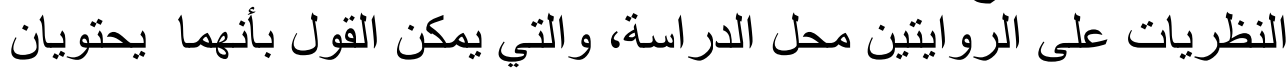
على العديد من الصور المختلفة للواقعية السحرية بما فيهما من خيال و عجائب وخروج عن المألوف عن طريق الدين والاحلام و الرجوع الى الى

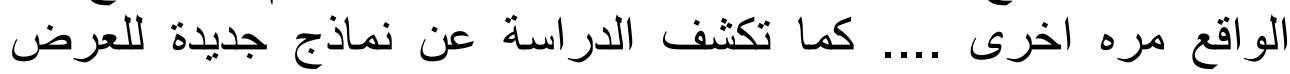
الروائي في صورة خيالبة بعيدة عن التقليد. 
Le réalisme magique dans La jument verte de Marcel Aymé et Nawat Alkarm de Nagwa Chaban Dr. Ahmed Moawad Abd-Elhadi

Le réalisme magique dans La jument verte de Marcel Aymé

\section{et Nawat Alkarm de Nagwa Chaban}

Notre réflexion porte plus précisément sur la notion du réalisme magique à travers les deux romans du corpus. Dans cette recherche, nous essayerons d'appliquer les théories concernant le réalisme magique. Ainsi, les deux romans choisis nous permettent-ils d'analyser les divers aspects du réalisme magique: le merveilleux, la religion et le rêve.

Daniel-Henri Pageaux indique que l'apparition du réalisme magique " coöncide avec l'essor du Surréalisme qui ne tolérait guère d'autres "ismes" à ses côtés et se montrait totalement hostile au genre romanesque. [...] du coup, c'est tout un pan de la création française qui a du mal à se faire reconnaître, à se situer, celle qui va du "magicien" Cocteau à Marcel Aymé qu'il est commode de réduire à un moraliste féroce. ${ }^{\prime \prime}$ Le réalisme magique ne représente pas une école ou un courant, mais une conception du monde. Le réalisme magique est une conception esthétique dont la fortune ne s'est étendue que très légèrement. En effet, la coïncidence avec le courant surréaliste se présente de surcroît comme une embûche à l'adoption du terme. En Amérique latine, la fonction excessive de l'étiquette par la critique a engendré une déception réelle chez les écrivains, dont Carlos Fuentes: " Gabriel Garcia Marquez a réussi à fermer, de sa cicatrice historique, les plaies saignantes $d u$ soi-disant "réalisme magique" qui, inventé par Alejo Carpentier, a été appliqué comme une étiquette indifférenciée à de trop nombreux romanciers hispano-américains, bien qu'il soit devenu la marque d'un seul: Gabriel Garcia Marquez" ${ }^{2}$

(1)- PAGEAUX, Daniel-Henri (2005)-'Préface", Charles W. Scheel" Réalisme magique et réalisme merveilleux: des théories aux poétique, Paris, L'Harmattan, p. 8

(2)- FUENTES, Carlos (1992)- le sourire d'Erasme: Epopée, utopie et mythe dans le roman hispanoaméricain, Paris, Gallimard(Le Messager), p. 28. 
La beauté réaliste magique selon Roh "demeure intéressée par la problématique des niveaux métadiégétiques qui inventent une frontière entre le réel et la fiction" ${ }^{1}$. Dans Le Rideau, Milan Kundera peint la nature d'un tel regard: "Plus on observe attentivement, obstinément une réalité, mieux on comprend qu'elle ne répond pas à l'idée que tout le monde s'en fait; sous un long regard de Kafka, elle se révèle de plus en plus déraisonnable, donc irraisonnable, donc invraisemblable. C'est ce regard avide posé longuement sur le monde réel qui a conduit Kafka, et d'autres grands romanciers après lui, par-delà la frontière du vraisemblable. ${ }^{\prime 2}$ L'œuvre de Kafka est comme l'une des meilleures manifestations du réalisme magique. La citation de Kundera dévoile que l'observation continue du réel permet au créateur réaliste magique d'apercevoir le déraisonnable.

Selon la différenciation de Chiampi;" 3 le réalisme magique peindrait la réalité tandis que le réalisme merveilleux serait la réalité, c'est-à-dire que le monde hors-texte est identique au monde du texte, ses surnaturels y compris. Chiampi présente une première étude narratologique de texte réaliste magique en observant la relation narrateur-narrataire. Il utilise "la notion de narrateur et celle de narrataire selon la théorie narratologique de Gérard Genette dans Nouveau discours du récit"4. Mais Jean Weisgerber offre une autre définition du réalisme magique dans les genres littéraires, cette définition devient aujourd'hui ce qu'il est convenu d'appeler le réalisme magique:" qui n'est ni un mouvement d'avant-garde, ni même une école, mais un simple courant littéraire groupant des écrivains isolés et qui s'insère

(1)- ROH, Franz(1925)- Nach-expressionismus (Magischer Realismus: Probleme der neuesten europäischen Malerei, Leipzig, Klinkhardt \& Biermann, (traduction française avec présentation et notes par Jean Reubrez Postexpressionnisme Réalisme magique Problèmes de la peinture européenne la plus récente, Dijon Les presses du réel 2013) p. 8

(2)- KUNDER, Milan- (2005)- Le Rideau- Paris, Gallimard, pp. 90-91.

(5)- GENETTE, G., (1983)- Nouveau discours du récit, Seuil, Poétique

(4)- ibid. 
Le réalisme magique dans La jument verte de Marcel Aymé et Nawat Alkarm de Nagwa Chaban Dr. Ahmed Moawad Abd-Elhadi

dans le réalisme élargi du XXe siècle [...] Sa magie s'oppose aux postulats sur la réalité, la perception et la logique en honneur au, milieu du siècle dernier, et jugés désormais trop étroits"l.

A ce point distinct, l'essor de Pageaux commence à être un peu plus exposé. Selon lui, l'histoire, dans la narration réaliste merveilleuse, "est l'objet d'une vision exaltée, traduite en un langage d'une poésie tendant au lyrisme" ${ }^{\prime 2}$ Également, le document est précisé par la très grande proximité sensible de l'auteur, du sujet et de l'objet. Dans cette étude, le réalisme magique, selon le modèle de Chanady"13," est exemplifié en contre partie par La jument verte de Marcel Aymé."14 Scheel indique qu'il ne souhaite pas préciser surtout la notion d'exaltation "afin de ne pas encombrer ou limiter inutilement son modèle. ${ }^{5}$ Bref, Le réalisme magique est une appellation utilisée pour rendre compte des productions où des éléments aperçus et arrangés dans La Jument verte et Nawat Elkarm comme magiques, surnaturels et irrationnels, surgissant dans un environnement défini comme «réaliste », à savoir un cadre historique, géographique et culturel vraisemblable. Pour le monde arabe, l'apparition du réalisme magique était depuis l'unique composition des contes " de Mille et Une nuit" puis les traditions populaires dès 1960 .

(1)- WEISGERBER, Jean-(1987)-(dir), Le Réalisme magique,:roman, peinture et cinéma, "La locution et le concept", Bruxelles, L'âge d'Homme, P: 27

(2)- PAGEAUX, Daniel-Henri op.cit., p. 116.

(3)- CHANADY,Amaryll- (1999)- Entre inclusion et exclusion, Paris, Honoré champion. P:

(4)- GIGUERE,Marielle- op.cit, P. 22.

(5)- SCHEEL, Charles W-(2005)- Réalisme magique et réalisme merveilleux-Des théories aux poétiques, Paris, L'Harmattan, p.117.

April 2018

Vol. 49 
La production littéraire de Marcel Aymé et Nagwa Chaban est influencée par l'inclusion de l'extraordinaire dans la fiction, c'est parce qu'ils ont reçu une culture traditionnelle dans laquelle les croyances religieuses et les fantasmes sont caractéristiques d'une situation sociale. Ces deux romanciers font intégrer dans le cadre réaliste des événements magiques comme des embûches magiques. Les personnages de La jument verte et Nawat Alkarm acceptent ces éléments surnaturels sans éprouver d'inquiétante étrangeté. Par ailleurs, dans le réalisme magique, le vocabulaire est emprunté aux mythes et aux croyances d'une culture spécifique. Dans cette recherche, nous avons emprunté le terme "les embûches magiques" qui signifie les aspects du réalisme magique chez Giguère, cité dans son ouvrage : Julien Gracq et le réalisme magique ${ }^{(1)}$. Les embûches magiques indiquent les figures magiques qui se trouvent dans La Jument verte et Nawat Alkarm, qu'il s'agit soit d'images merveilleuses ou hors du commun, comme les paroles de la jument, soit d'images religieuses ou rêves.

La jument verte est un roman réaliste qui raconte la société française au $\mathrm{XIX}^{\mathrm{è}}$ siècle. L'action se déroule au village de Claquebue sur une trentaine d'années à la campagne. C'est une histoire de la famille Haudouin et de ses rapports avec les autres familles du village de Claquebue. Elle se déroule sous le regard et les commentaires de la jument verte immortalisée dans un tableau accroché au mur de la salle à manger, ce qui permet au lecteur de reconnaître les caractères des différents protagonistes selon plusieurs perspectives. Pour ce qui est des événements du roman Nawat Alkarm,ils se passent à Damiette, la terre natale de la romancière au début du $\mathrm{XVI}^{\mathrm{e}}$ siècle.

(1)- GIGUERE, Marielle, Julien Gracq et le réalisme magique, Mémoire soumis à l'Université McGill en vue de l'obtention du grade de M. A. en langue et littérature françaises, Montréal, (2007) p. 7

April 2018 
Le réalisme magique dans La jument verte de Marcel Aymé et Nawat Alkarm de Nagwa Chaban Dr. Ahmed Moawad Abd-Elhadi

En mentionnant les toponymes, Claquebue et Damiette, les deux romanciers cherchent à atteindre" un ancrage historique [...] visant à construire le simulacre d'un référent externe et à produire l'effet de sens réalité." ${ }^{1} \mathrm{Ce}$ qui importe ici ce ne sont pas les noms de lieu en eux-mêmes, mais plutôt leur valeur significative. Les deux villes natales sont des villes maritimes qui se donnent à une grande surface de l'eau. Ajoutons que le contexte historique dans les deux romans est proche; La jument verte est essentiellement une étude de la morale sexuelle et des comportements des habitants d'un petit village français du XIXe siècle et le roman Nawata Alkarm raconte l'histoire de la famille de Bassal à la fin de l'ère des Mamelouks et le début de l'ère des Ottomans.

Chaque analyse reste sensible aux modalités du genre et vise à mettre en évidence sa complexité en abordant des problématiques différentes. Dans cette recherche, nous remarquons que Marcel Aymé et Nagwa Chaban ont suivre la même stratégie. Chacun d'eux raconte une époque précédant sa naissance pour pouvoir résister à son présent amer. Marcel Aymé est un écrivain français, né à Joigny le 29 mars 1902 et mort à Paris. Il offre à travers son roman une image de la société au $\mathrm{XIX}^{\mathrm{e}}$ siècle. Il est resté très attaché à sa région d'origine, la Franche-Comté qui lui a servi de décor dans La Jument verte. C'est de ce monde-là qu'il s'est inspiré pour décrire les vives passions politiques ou religieuses du monde rural: «Marcel Aymé a fini par occuper un ministère parfaitement reconnu: celui de l'ironie politique et de l'inconfort intellectuel. "2 Quant à Nagwa Chaban, elle est une romancière égyptienne, née à Damiette. Elle présente à travers son roman une description de la

(1)- GREIMAS (A. J) et COURTES (J), Dictionnaire raisonné de la théorie du langage, Hachette, Paris, 1979, p. 133.

(2)- Dictionnaire historique, thématique et technique des littératures, (1985)- vol. I, p. 135, Larousse, (ISBN 978-2-03-508301-2). 
société du $\mathrm{XVI}^{\mathrm{e}}$ siècle. Elle est restée fidèle à sa ville natale, qui lui a servi de décor pour Nawat Elkarm.

La jument verte est un ouvrage plein d'aspects merveilleux qui sont considérés comme des embûches magiques. Nous pouvons relever à l'appui la citation suivante de notre corpus: "Au village de Claquebue naquit un jour une jument verte, non pas de ce vert pisseux qui accompagne la décrépitude chez les carnes de poil blanc, mais d'un joli vert de jade.(...) - Ce n'est pas possible, disait-il, j'aurais trop de chance." ${ }^{1}$ Cette citation est l'exposition de la Jument verte qui annonce un roman réaliste en citant l'espace et le temps; un village qui se trouve à la campagne française, à Claquebue, village du Jura franc-comtois, où Marcel Aymé a passé son enfance. Un homme s'aperçoit un jour qu'il possède une jument verte, sa couleur de jade, qui fera la fortune de son propriétaire, le maquignon Jules Haudouin. Cela se passe vers 1860. L'auteur expose réel et magique, possible et impossible, présentant des thèmes de la réalité; une réalité chez tout le monde" la jument" à l'interaction de l'irréel en employant l'expression " la jument verte". Sa couleur est un événement drôle et irréel parce qu'il n'y a pas de cheval vert et le symbolisme de la couleur" est presque toujours en liaison avec des passions sexuelles» ${ }^{2}$ Ce cheval est d'un joli vert de jade, sa couleur signifie qu'il s'éveille chez un cultivateur maquignon qui n'a jamais été récompensé d'être rusé. C'est une grande nouveauté: naissance d'une jument verte qui n'avait point de précédent connu! La jument verte paraît remarquable car dans ce village, il n'arrivait jamais. Enfin, "Jules Haudouin parut sur le seuil de l'écurie. Hilare, les mains sanglants, il confirma:-Elle est verte

(1)- AYME, Marcel, (1933)- La jument verte, Paris, Gallimard, pp. 8,9

(2)- KURTH, Hanns, (1977). Dictionnaire des rêves de A à Z, France Loisirs, Paris, boulevard de Grenelle, p.142. 
Le réalisme magique dans La jument verte de Marcel Aymé et Nawat Alkarm de Nagwa Chaban

Dr. Ahmed Moawad Abd-Elhadi

comme une pomme! $\gg^{1}$

Les événements du roman Nawat Elkarm sont pleins de couleurs merveilleuses qui sont considérées comme des embûches magiques. Nous pouvons relever la citation suivante:

" À cette époque, à côté de Damiette, les tempêtes d'Alkarm_arrachaient un vieil arbre de citron, cet arbre lance de son corps des éclats aiguilliers qui pénétraient les corps des gens et les pauvres dans leurs maisons modestes." $2 "$ (Nous traduisons)

C'est une citation qui située à l'incipit de Nawat Elkarm, annonce un roman réaliste en mentionnant le temps et l'espace: un village qui se trouve près de Damiette, où Nagwa Chaban a passé son enfance. En effet, si Marcel Aymé a utilisé dans son roman un animal" la jument verte", la romancière de Nawata Alkarm a utilisé l'arbre pour exposer un événement qui est réel et magique à la fois où elle a brouillé les limites entre le possible et l'impossible, présentant des thèmes de la réalité; une vérité chez tout le monde" l'arbre" à l'interaction de l'irréel en employant l'expression " cet arbre lance de son corps des éclats aiguilliers ". Événement drôle et irréel:il n'y a pas d'arbre qui lance des éclats!

Dans La jument verte, le surnaturel est une occurrence ordinaire. L'ordre logique étant ainsi retourné, ce sont rarement les principes du réel qui sont considérés magiques. La jument verte est naturellement marquée par des thèmes subversifs qui relatent une interprétation sur la situation sociale. Dès son titre, La jument verte signale son lien au surnaturel.

"A quelque temps de là, un professeur du collège impérial de Saint-Margelon, vint voir la jument verte. Il demeura éberlué et en écrivit à

(1)- La jument verte, P. 7.

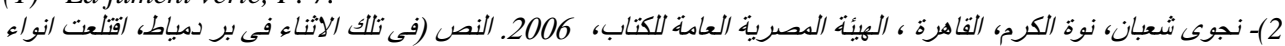

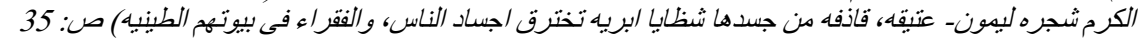
April 2018 55 Vol. 49 
l'Académie. Un savant illustre, décoré jusqu'à droite, déclara qu'il s'agissait d'une fumisterie. "J'ai soixante-seize ans, dit-il, et je n'ai lu nulle part qu'il ait existé des juments vertes "l

Dans cette citation, l'auteur présente une autre allusion à la jument verte qui est considérée comme un aspect du réalisme magique en se demandant: « Est-ce que cet animal est réel ou non?» Il expose l'état d'hésitation d'un professeur du collège impérial qui vient pour voir cette jument verte, avec un autre savant illustre, en déclarant qu'il s'agissait d'une fumisterie, en disant qu'il n'a lu nulle part qu'il ait existé des juments vertes. C'est-à-dire un cas de l'hésitation entre le possible et l'impossible.

Pour dire qu'un texte est réaliste magique selon Todorov, il faut que le texte oblige le lecteur à considérer le monde des personnages comme:" un monde de personnes vivantes et à hésiter entre une explication naturelle et une explication surnaturelle des événements évoqués"2. L'expression "courant littéraire" méritera d'être réexaminé parce qu'elle " donne l'émotion d'un groupe d'unités mélangée autour d'une même opinion, alors que le réalisme magique est davantage une esthétique que les auteurs exploitent occasionnellement."(3) A travers les événements de Nawata Alkarm, nous hésitons entre le réalisme et l'imaginaire. Le surnaturel s'est réalisé à travers la procuration du rationnel et de l'irrationnel. Ce type d'écriture crée le fusionnement et la surprise chez le lecteur. Nous trouvons que Nagwa Chaban a achevé cette création magique par son langage médicinal: nous remarquons qu'elle a utilisé l'entassement qui permet de développer sa phrase en s'appuyant particulièrement sur la relation terminologique pour créer un ensemble lié, destiné à faire une liste des objets contre la magie dans la citation

(1)- La Jument verte, P. 10

(2)- TODOROV, Tzvetan, Introduction à la littérature poétique- Paris, Ed. du Seuil, (Coll. « Points/Essais), (1976), p. 37

(3)- TROUSSON,Raymond, "Du fantastique et du merveilleux au réalisme magique?",Le Réalisme magique-Roman-Peinture-Cinéma, Jean Weisgerber, (1987), p. 38. 
Le réalisme magique dans La jument verte de Marcel Aymé et Nawat Alkarm de Nagwa Chaban Dr. Ahmed Moawad Abd-Elhadi

suivante:

"La réponse de Laîl à son mari qui a montré les portes des maisons à Rhodes, il y a en haut de ces portes; un tas d'ails. Il lui a dit qu'ils ont également mis une couronne de lauriers, une noix de sorcière, des cailloux en trous comme amulette, des petites boules de verre vert s'accrochées sur les fenêtres, des clarines du cheval ou une pièce métallique placée sous les sabots des chevaux, Enterrement les couteaux sous les sols des maisons." 1 (Nous traduisons)

Dans cette citation, la romancière présente un autre symbole qui est observé comme une forme du surnaturel en se demandant des recettes magiques, indiquées là-haut, qui portent des noms d'une même classe à l'intérieur d'une phrase. L'entassement de plusieurs noms donne le sentiment d'étrangeté. Cette accumulation par juxtaposition et coordination met en valeur les matériaux du langage thérapeutique et magique. Elle permet de rendre une idée plus frappante, de faire naître une impression de suspense, de créer un effet de profusion et de réalisme magique.

Depuis plus de quarante ans, le réalisme magique, type charmant et étonnant qui vise à l'assemblage du réel et du fantastique, connaît un succès brillant. De toute certitude, la renommée de l'œuvre de Marcel Aymé confirme le succès du genre et est propagée dans le monde entier. Le réalisme magique brouille les limites entre le possible et l'impossible présentant ainsi des variétés, généralement antithétiques et thèmes de la réalité:" Quant à Honoré, son père lui donnait sa malédiction une fois par semaine, et cela n'allait jamais sans de grands coups

\footnotetext{
1) - نجوى شعبان , نوة الكرم، النصا:( كان رد ليل على زوجها الذى يشبر الى ابواب البيوت فى رودس وقد علتها حزم من الثُّم.

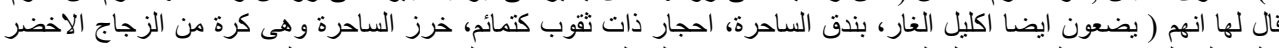

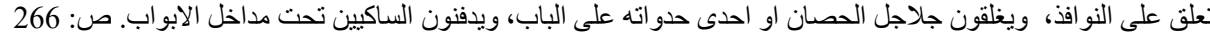
April 2018 
de gueule de part et d'autre." I

Dans cette embûche magique, le romancier utilise la métaphore en exposant que la malédiction est un cadeau d'Haudouin à son fils, une fois par semaine. C'est une merveille car la malédiction n'est pas transportée avec l'héritage du père à son fils, l'auteur cite cette expression pour assurer la pauvreté de cette famille, pourtant, le fils Honoré n'est pas un paresseux, ni un révolté, au contraire "c'est un bon fils comme il était bon père et bon époux; mais sa seule présence à Claquebue représentait un danger permanent pour les intérêts de sa maison.» ${ }^{2}$ Relevons aussi cette citation qui affirme le surna turel:

" Le père Haudouin, qui n'avait jamais été malade, se coucha un après-midi et mourut en une semaine. On l'enterra à côté de sa femme, et Ferdinand leur fit faire à tous deux de belles grosses pierres tombales en marbre noir, telles qu'on n'en avait jamais vu à Claquebue. Les pauvres morts d'à côté, sous leurs petits bourrelets de terre, en étaient attristés dans les nuits. ${ }^{13}$

Dans cette citation, l'écrivain rappelle l'embûche magique qui est considérée comme un aspect du réalisme magique en citant que le texte se caractérise par l'existence d'éléments paranormaux, d'événements d'ordre surnaturel. L'auteur présente une image surnaturelle des morts dans leurs tombes comme s'ils peuvent parler ensemble dans leurs tombes. Il expose que les pauvres morts sont très tristes car ils demeurent dans des tombes en petits bourrelets de terre tandis que le père Haudouin et sa femme séjournent dans un tombe construit de belles grosses pierres tombales en marbre noir comme un palais. C'est ainsi que, le surnaturel dans le réalisme magique est énoncé de façon

(1)- La jument verte, P. 27

(2)- Loc.cit.

(3)- Ibid. P. 29. 
Le réalisme magique dans La jument verte de Marcel Aymé et Nawat Alkarm de Nagwa Chaban Dr. Ahmed Moawad Abd-Elhadi

arrangée. Il y a donc un code du surnaturel de magie et un code du réel, le surnaturel se juxtapose au réel de façon tout à fait naturelle. Telle est une image d'hésitation entre la vérité et la fiction.

Ajoutons qu'Amounette a aussi ses recettes médicinales pour faire guérir "Lail", l'endormir et atténuer son mal de tête. Le vocabulaire local peut donc composer les ingrédients de sa recette: "Une dose qui pèse deux grains de poudre de cinname avec de l'eau de la laitue" ${ }^{1}$ Notons qu'Amounette et le père Haudouin possèdent une connaissance profonde du monde physique et du monde Invisible. Le langage de ses deux personnages leur donne une force et une supériorité sur leur communauté qui les appelle sorciers. Le pouvoir du vocabulaire magique s'intègre et se défile dans le corps des événements réalistes des deux romans pour créer un réalisme magique. Marcel Aymé se caractérise aussi par l'étendue de son bagage culturel, par l'importance du jeu intertextuel qui sous-tend aisément les fictions réalistes magiques: "Il (Honoré) sifflait sur la plaine en poussant sa charrue, puis s'arrêtait pour pisser, reprenait, crachait à gauche, chantait, parlait à ses boufs, les caressait à lisse et à contre-poil, ... $\rangle^{2}$ L'auteur présente cette citation pour indiquer qu'on ne peut pas préjuger qu'il partage les croyances absurdes de ses acteurs. Il présente cette étiquette magique qui est considérée comme un aspect du réalisme magique en citant Honoré qui sifflait à la campagne en poussant sa machine, parlant à ses animaux, et s'émerveillant qu'il fît aussi bon vivre. C'est la troisième condition qui exige que "le lecteur adopte une certaine attitude à l'égard du texte"13.

La jument verte et Nawat Alkarm se composent de la

النص "شعبرتين من مسحوق الكافور مع ماء الخس" ص: 116 (Nawat Elkarm (1)-Lajerm )

(2)-La jument verte, $p 54$.

(3)- TODOROV,T op.cit, p. 38.

April 2018 
réalité culturelle où le texte magique produit des univers fictifs qui ont un rapport avec le monde réel. Le lien entre le genre et le contexte social dont il découle est le fondement du réalisme magique. Les théoriciens du réalisme magique, comme Chanady ${ }^{1}$ et Scheel, $^{2}$ soulignent que le lecteur reconnait un contexte socioculturel dans le texte. Donc, Les deux romans sont deux types qui privilégient une lecture des aspects thématiques et idéologiques représentant la situation culturelle et en même temps, l'hésitation se trouve représentée " elle devient un des thèmes de l'ouvre $»^{3}$. La place du fantastique appartient de ce fait au lecteur, variant selon l'augmentation du trouble qu'il ressent. De ce fait, le fantastique est un genre dans lequel le réalisme narré "exige que le narrataire fasse la passion entre son univers et celui narré dans le récit. Il n'est donc pas question de symbole ni de métaphore, ni d'interprétation "poétique" ${ }^{\text {". }}$ Le réalisme magique se distingue du surréalisme, tandis que le fantastique se distingue de l'absurde. Le surnaturel peut être perçu comme thème dans le fantastique puisqu'il est opposé au cadre réaliste tel qu'il est établi par le texte, mais le surnaturel est accepté en partie dans le réalisme magique. C'est ainsi que le masque de $L a$ jument verte et Nawat Alkarm permet de contourner la censure exercée sur les études sociohistoriques comme le réalisme magique qui "répond aux enjeux de son temps: une recherche du sacré et des traditions anciennes face à un monde matérialiste"s

Dans Nawat Elkarm, l'usage d'expressions thérapeutiques est assimilé dans les recettes magiques pour résoudre un problème sociohistorique soit psychique soit physique; voici par exemple

(1)- CHANADY,Amaryll, Entre inclusion et exclusion- Paris, Honoré champion, (1999).

(2)- SCHEEL, Charles W, op.cit, P.256

(3)- TODOROV, T op.cit, p. 37

(4)- Ibid., p. 38.

(5)- ROUSSOS, Katherine, Décoloniser l'imaginaire:leréalisme magique chez Maryse Condé, Sylvie Germain etMarie Ndiaye, Paris, L'Harmattan, (2007) pp.17-19. 
Le réalisme magique dans La jument verte de Marcel Aymé et Nawat Alkarm de Nagwa Chaban Dr. Ahmed Moawad Abd-Elhadi

la recette magique de la femme de [Elmegabaraty] ${ }^{1}$ (la personne qui éclisse les membres fracturés) qui croit que son mari est tombé en proie à un acte magique. Comme dans $L a$ jument verte la recette vient sur la langue d'une personne expérimentée :"l'amour était comme le vin d'un clos familial". La recette magique se compose de la sueur de la mariée avec de la farine. "La pétrisseuse des muscles" ${ }^{2}$ accumule aussi sa recette magique: les ongles des mains et des pieds, un peu de cheveux, de la farine et un œuf. Elle fait tous ces ingrédients avec la pâte déstinée au mari "afin de le garder toute la vie "3 Lail, de son coté, convoqua son amie [Elghagariya]" 4 la bohémienne" pour qu'elle lui prépare une recette magique lui facilitant de réaliser son désir: faire l'amour avec Elmegabaraty. Le choix du moment est indispensable et se considère comme un outil étranger au réalisme magique. " Le vendredi à minuit" 5 avec son mot magique [Bedouh]"6," un talisman particulier et le "vin de palme"7 En effet, l'usage du vocabulaire régional informe l'effort du lecteur. Dans ces citations, nous remarquons que le style de la romancière est débarrassé de toute ambiguïté.

Nous pouvons citer l'embûche magique suivante dans la maison d'Honoré, la jument verte raconte cette confusion en disant:" l'amour était comme le vin d'un clos familial; on le buvait chacun dans son verre, mais il procurait une ivresse que le frère pouvait reconnaître chez son frère, le père chez son fils, et qui se répandait en chansons du silence. ${ }^{18}$ Dans cette citation, il y a une autre confusion entre la vérité et l'imaginaire, ce

(1 ) Dans Nawata Elkarm, le terme " المجبر انىى [ Almegabaraty] signifie la personne qui soigne les fractures et non pas médecin spécialiste d'orthopédie rebouteur rebouteux.

(2 )- Ibid, le terme " " المدلكة 105

(3 )- Ibid, يظل خاضعا محبا للك مقعيا عند قدميك " N.A p. 106.

(4) - Ibid, الغجرية " N.A p. 103.

(5 ) - Ibid, " N.A p. 103.

(6 ) - Lبوح " Le mot " [Bedouh] signifie en arabe l'amour et la cordialité mais il est écrit inversement d'une manière magique.

(7) Nawat Elkarm, " طلسم خاص و "عرق البلح " N.A p. 103.

(8)- La jument verte. 149 
réalisme magique - celui qui met en scène une magie qui découle à travers le style de Marcel Aymé. L'usage de ces mots exprime l'état d'ivresse qui indique le voile délicat entre le réel et l'imaginaire. Les deux romanciers sont sûrement conscients de l'influence du vin pour achever les actes magiques et ses effets thérapeutiques. Dans la jument verte, le style est un élément essentiel: c'est grâce à une influence fine du matériau langagier que le créateur arrive à dissoudre l'un dans l'autre, le code réaliste et le code mystérieux "dans un discours extraordinairement poétique à tous les niveaux de la narration"l

Les paroles de la jument verte dans le roman de Marcel Aymé sont considérées comme un type de merveilleux parce que les animaux ne parlent pas et son propos est exceptionnel, mais l'auteur consacre un espace à la jument verte en racontant les secrets privés des familles dans ce village. La jument verte dit:

" J'ai connu quatre générations de Haudouin, la première à son âge mûr, la dernière à son matin. Pendant soixante-dix ans, j'ai vu les Haudouin à l'ouvre d'amour, chacun y apportant les ressources d'un tempérament original..." 2

Dans cette citation, il y a un plan magique à travers les propos de la jument verte où elle narre qu'elle a suivi quatre générations de la même famille. En effet, c'est une image d'hésitation entre le possible et l'impossible, mais l'auteur veut envoyer un message au lecteur par la jument verte qui découvre les secrets des maisons à l'œuvre d'amour dans la recherche du plaisir. C'est un mystère bien au-dessus de la connaissance de la jument verte: " Il est donc impossible d'imaginer dans le détail le mécanisme de transmission. Les parents enseignent leurs enfants dans une certaine manière de faire l'amour,.." 3

(1)- PAGEAUX, Daniel-Henri, Op.cit. p. 113

(2)- La jument verte, p 18.

(3)- Ibid, $p 19$.

April 2018 
Le réalisme magique dans La jument verte de Marcel Aymé et Nawat Alkarm de Nagwa Chaban Dr. Ahmed Moawad Abd-Elhadi

A travers ses propos, la jument verte, avoue que parmi les membres de la famille de Haudouin, elle a aimé Honoré Haudoin, le ca det qui a 45 ans, et a épousé contre la volonté de son père une fille mince aux yeux noirs, Adélaïde Mouchet âgée de 40 ans au moment de l'action,:" Entre tous les Haudouin de Claquebue et d'ailleurs, Honoré eut toujours ma préférence(...) J'ai découvert, chez cet homme tendre et rieur, le secret d'un érotisme spacieux qui trouvait ses meilleures satisfactions hors de la réalité. "l Hellens ajoute en outre que " l'imagination poétique est le principal moteur du fantastique réel". ${ }^{2}$ Il indique comment l'application et la lenteur peuvent faire provenir un merveilleux caché dans le réel:" C'est ici la place d'une sorte de fantastique continu ou de réalisme envoûtant; une sorte de transfiguration momentanée par petites touches, de l'objet qui éclate. ${ }^{\prime 3}$. Marcel Aymé présente une autre allusion à la jument verte qui est considérée comme un aspect du réalisme magique en citant les paroles de la jument verte: "... pour la majorité des hommes de Claquebue; à l'âge de l'adolescence poussée, ils se séparaient de leurs habitudes d'amours ingénues, turbulentes, impudiques, et prenaient le chemin de choisir une femme et de se limiter en toutes façons. " ${ }^{4}$ Dans cette citation, l'auteur présente la jument verte comme un personnage principal et lui donne l'occasion de raconter les événements comme un narrateur. C'est un discours extraordinaire qui est considéré comme une transformation temporaire par petite touche. Sa parole merveilleuse est précisée par un discours extraordinairement et par la fusion de deux règles opposées qui le traitent, ce sont le réel et la fiction.

La jument verte finit ses paroles au dernier propos, citant

(1)- Ibid, pp.52, 53

(2)- HELLENS, Franz- Le fantastique réel, Bruxelles, Sodi, (1967), p. 57

(3)- Ibid, p. 70

(4)- La jument verte, p.121.

April 2018 
que les écrivains narrent les événements de la société et en même temps ils ne peuvent pas changer la morale en indiquant un conseil d'amour durable: "Les romanciers sont des gens à la tête légère, ils racontent des histoires, et la morale y va comme elle peut. Je le dis sans orgueil : il est bien heureux qu'une jument verte se soit trouvée là pour tirer de ce roman un robuste et honnête enseignement...

Dans cette citation, il y a une étiquette magique qui déclare que la jument verte critique la société française en citant la famille d'Haudouin comme un aspect de la société dans cette époque. Ajoutons que la jument verte n'est pas un véritable animal mais il incarne la voix de l'auteur qui fuit de critiquer la société directement. Nous remarquons qu'il y a glissement un fil entre réel et magique chez Marcel Aymé et Nagwa Chaban, ce fil déchiffre la conscience de deux romanciers qui balancent leur écriture entre le réel et la magie afin de pousser le lecteur à entrer dans leur monde particulier du réalisme magique.

Après avoir discuté le merveilleux dans les deux romans et les propos de la jument verte comme un aspect du réalisme magique chez Marcel Aymé seulement, nous passons à un autre type d'embûches magiques qui apparaissent comme des croyances religieuses. L'évocation de la Jument verte et l'arbre qui lance de son corps des éclats aiguilliers sont déjà les premières allusions à l'objection, car ils renvoient non seulement à une célébration de la sorcellerie, mais également à une tradition ancrée dans le religieux. La Jument verte présente toutes les caractéristiques du genre, à une époque où le réalisme magique n'avait pour cadre théorique que des notions imaginaires. Pouvons-nous relever à l'appui la citation suivante de notre corpus:

"Un moment, il (Haudouin) demeura immobile et 
Le réalisme magique dans La jument verte de Marcel Aymé et Nawat Alkarm de Nagwa Chaban

Dr. Ahmed Moawad Abd-Elhadi

coi, comme se dérobant à un péril, puis l'obscurité lui rendit l'inspiration; j'entendis le souffle rauque du vieillard et le halètement complaisant de la servante. Cette aversion, mystique pour le plaisir des yeux, cette croyance obscurément enracinée que la vision du péché est plus abominable que le péché lui-même... "1

Dans cette citation, Marcel Aymé montre une opposition entre le désir (qui attire Haudouin au monde magique) et la religion (qui interdit d'échapper de la réalité) selon le curé du village qui indique au monde rural que la vision du péché est plus grave que l'acte de faire ce péché. C'est une croyance chez les gens du village qui croient aux paroles du curé, cet homme qui parle au nom de Dieu.

Quant à Nawat Elkarm, nous remarquons que les expressions religieuses tirées de la bible peuvent composer une recette magique au moment de l'accouchement d'une amie: la danseuse juive Bissi tire une feuille de papier magique de son "sac de toile". ${ }^{2}$ Elle a mis cette feuille de papier sous sa langue et a commencé à réciter des mots de la Bible. Les femmes se demandent d': "Une feuille comporte quatre lettres $(A, M, F, T)$, c'est un carré magique qui comprend les quatre lettres des noms et versets bibliques" ${ }^{\prime 3}$ L'étrangeté de cette situation magique qui relève des croyances religieuses de la danseuse juive Bissi provient de cette recette transmise à ses amies.

Parfois, le langage magique montre le caractère irrationnel qui s'attache à une croyance. Dans Nawat Elkarm, Lail prépare une recette magique pour que ses frères jumeaux aient une très bonne santé tout le long de leur vie. Un commerçant soufi lui

(1)- Ibid, p. 20

(2) Nawat Elkarm, "بقنه" " N.A p. 229.

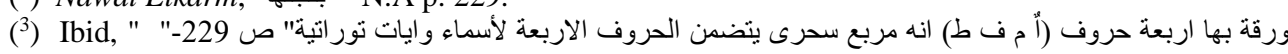
230

April 2018 
conseille de "farcir une datte d'une partie de placenta $d u$ nouveau-né": ${ }^{1}$ Le surnaturel admis comme une réalité à part entière vient à l'extérieur d'un personnage secondaire mais il possède une expérience religieuse. Dans Nawat Alkarm, les personnages créent un catalogue des objets pour se protéger contre l'inconnu ou le dommage des vêtements ou des matériaux. Le lecteur observe que Elmatarawy, un personnage bizarre, croit bien à la superstition. Il croit que certaines recettes peuvent entraîner de bonnes conséquences contre la magie. Il donne à Amounette "deux morceaux de cuticule pour protéger les vêtements contre les anobidés et la mite" ${ }^{2}$ Ajoutons une autre magie blanche pour traiter sa maladie qui consiste à: " plonger un bout (partie extrême) pointu d'une palme dans l'estomac d'une statue d'en cire à l'allure de Sinaniya" ${ }^{3}$ (Nous traduisons)

L'exposé de la magie blanche dans les deux romans met en évidence les croyances de l'ère historique chez des personnages pour des raisons culturelles et intellectuelles. Les deux romanciers décrivent deux mondes d'êtres à la fois visibles et invisibles. Pour les personnages faibles dans Nawat Elkarm, les hommes de religion possèdent la réalité absolue. Sinaniya, embarrassée intellectuellement, tombe victime \{dans le piège\} d'un cheik soufi. La présence d'un homme religieux avec son style rompt l'opposition établie entre la réalité et la magie. Il existe aussi dans La jument verte, qui est pleine de contes, de "savoirs absolus", de mythes, de légendes populaires. Voici une autre embûche magique religieuse pour échapper de la réalité pénible au réalisme magique racontant le cas de Frédéric qui a perdu sa petite amie dans un accident: " Le pauvre garçon devient amoureux d'une jeune fille qui mourut dans un accident de chemin de fer. Il crut son cour brisé à jamais et voulut mettre

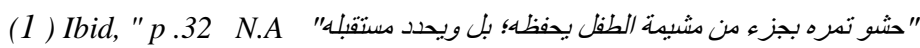

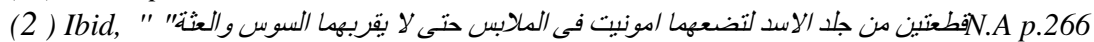

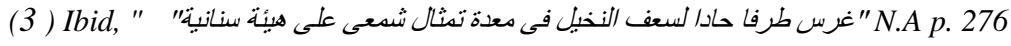

April 2018 
Le réalisme magique dans La jument verte de Marcel Aymé et Nawat Alkarm de Nagwa Chaban

Dr. Ahmed Moawad Abd-Elhadi

la chose en vers" ${ }^{1}$ Mais sa versification ne lui fait pas oublier ses douleurs, il cherche d'autre fuite, donc il décide d'entrer dans les ordres pour devenir un frère prêcheur. Alors, ce travail est comme un pan magique religieux : "Comme les rimes venaient mal, il cherche autre chose et pensa ne pouvoir moins faire que d'entrer dans les ordres. Il serait frère prêcheur. "2 C'est une vision particulière de sortir de ses douleurs et de son cas catastrophique.

Michel Dupui et Albert Mingelgrun énoncent trois caractéristiques, bien différentes de celles de Chanady, ces trois points participent à la définition du réalisme magique. Selon eux, pour qu'un texte soit admis comme réaliste magique, il faut: mettre en évidence une vision particulière, une manière hors du commun de regarder le monde, de sonder la réalité et de faire subir à son image une transfiguration" ${ }^{\prime 3}$. C'est clair avec les personnages de Nagwa Chaban qui manifestent un système culturel dynamique de représentation: "prépare de l'eau magique à l'aide d'un magicien qui a exorcisé une amulette sur l'eau. ${ }^{44} \mathrm{La}$ présence de l'eau est une expression réaliste mais l'ajout de l'adjectif "exorcisé" donne une force essentielle à l'expression et la présence d'un magicien a une valeur étrangère à la situation. Remarquons nous qu'il y a:" une distinction relative au statut de cette image du réel 'avant' et 'après' l'intervention de la magie artistique ${ }^{15}$

Dans Nawat Elkarm, la maîtrise du style magique médicinal a une valeur amusante à ses personnages. Ils craignent les hommes de religion qui utilisent la puissance des mots

(1)-La jument verte, p. 32

(2)-Ibid, p. 33

(3)-DUPUI, M et MINGELGRUN, A, "Pour une poétique du réalisme magique" Le réalisme magique-Roman-Peinture-Cinéma, Jean Weisgerber,éd, (1987) p. 219.

(4) Nawata Elkarm, p. 231 ("قامت بالقاء ماء عزم عليه الساحر بطلاسمه على بسطة السلم المطلة على غرقة سنانية

(5)-DUPUI, M et MINGELGRUN, A op.cit., p. 219

April 2018

67

Vol. 49 
prononcés. Les magiciens et les hommes de religion possèdent les mots mystiques pour garder le pouvoir exclusif que nous retrouvons dans les sociétés primitives. Un des points de la singularité chez Nagwa Chaban, c'est de mettre en évidence une vision particulière de sa parole magique et sa relation avec l'astrologie. Nagwa Chaban choque le lecteur par une pause qui s'échappe du contexte narratif du roman en mettant en évidence la relation entre la parole magique et l'astrologie :

"Ce que ne savait pas Lail -comme l'on lui dira bientôt- et que les corps célestes dans la Balance, le Sagittaire et le Verseau agissent rapidement contre elle à ce moment-là pour que les affaires fonctionnent contre elle" 1

(Traduit par le chercheur)

Nagwa Chaban introduit son roman par une mise en relief et la compression de la terminologie de l'astrologie comme "les corps célestes, Balance, Sagittaire, Verseau" à un moment donné manifesté par l'usage des lexiques de temps: " à ce moment-là ", " la derrière semaine du janvier" et la prédication de l'avenir de "Lail". C'est une manière hors du commun de regarder le monde. $L a$ romancière crée une relation magique triangulaire entre l'espace "L'astrologie", le temps "à un temps précis" et le destin de l'homme. Cette relation magique se base sur le vocabulaire de l'astrologie qui s'appuie sur les traditions et les croyances surnaturelles locales de la société égyptienne en évoquant la situation des corps célestes dans l'espace à une certaine date précise: " la derrière semaine du janvier" peut découvrir le sort de Lail, en tant qu'il résulte de forces célestes. La romancière présente cette pause pour attirer l'attention de son lecteur sur l'aspect magique de l'astrologie et l'avenir de Lail.

Encore une fois, la romancière nous offre son triangle magique: "Dans ce jour-là, le Scorpion sensuel s'intervient avec

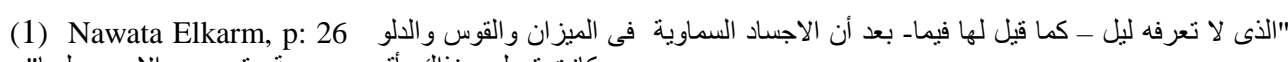
كانت تعمل حينذاك بأقصى سر عة حتى يسير الامور عليها" 
Le réalisme magique dans La jument verte de Marcel Aymé et Nawat Alkarm de Nagwa Chaban

Dr. Ahmed Moawad Abd-Elhadi

la Venus, ce qui rend les gens très sensuels, émotifs et agités" Cette citation magique d'astrologie est très significative: elle collectionne deux types de l'astrologie "le Scorpion sensuel" et " la Vénus" " la planète de l'amour"; ce qui affirme l'idée de magique chez les habitants de Damiette, qui étaient très sensibles. C'est une façon hors du commun d'examiner la société de la romancière qui crée une relation absurde de cause et de conséquence : à cause d'une idée déformée prétendant que le dernier jour de jugement se passerait prochainement. Toutefois, les indigents de la ville attendent ce jour avec impatience afin de se débarrasser de leurs souffrances physiques et morales. Cette relation illogique certifie la croyance religieuse qui s'attache à l'astrologie.

Ajoutons que la réalité religieuse se lie au surnaturel dans la société égyptienne à travers la mise en scène de l'Epiphanie" ${ }^{2}$ en sa relation avec la curie des maladies: "... c'est pour cela à l'Epiphanie précédente à 11 Tybi, je vous ai plongé tous dans une eau froide ... pour que vous auriez une bonne santé tous au long de la vie"3 Dans cette citation, l'embûche magique naît de deux termes qui symbolisent une réalité historique à propos de la religion chrétienne "l'Epiphanie"4 et sa date selon le calendrier copte en 11 "Tybi " et d'autre côté la puissance de l'eau froide de procurer aux nouveaux- nés une très bonne santé tout au long de leur vie. La romancière compose sa magie en ajoutant des expressions surnaturelles au fil de son écriture réaliste. Ces expressions colorent d'une façon précise l'univers magique de l'écriture chez Nagwa Chaban qui s'attache à présenter son triangle magique, elle expose sa parole magique dans la séquence

(1 ) Nawat Elkarm, فى هذا اليوم حدث تداخل بين برج العقرب الحسى وكوكب المحبة فينوس/الزهرة مما جعل الناسل

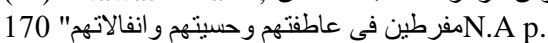

(2)- C'est la fête d'eau qui venue d'Orient où elle a été fixée au 6 janvie.

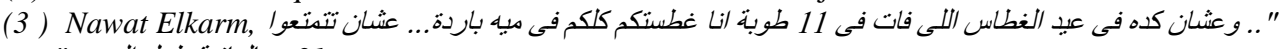

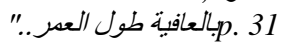

(4) Ibid, p. 31 " عيد الغطاس

April 2018 
suivante: " Au mois de Mésori, au moment d'entrer le soleil au Lion, ce temps de la promesse de crue et la fertilité, donc, à ce jour, Lays El Din est revenu. "l Elle commence sa phrase par la parole magique en citant un terme d'astrologie "Le Lion" et sa relation avec le mois de "Mésori", (qui se prolonge du 7 août au 5 septembre). La romancière crée une relation magique de quatre éléments; ce sont, une précision temporelle magique " le Lion" ; le second est un mois du calendrier copte le mois de Mésori; le troisième est la promesse de crue et la fertilité. Tous ces trois éléments nous annoncent le quatrième élément: le retour de Lais El Din de son voyage possédant de l'argent pour la première fois dans sa vie. Tous les termes magiques d'astrologie font subir à son image une transfiguration qui dévoile la croyance des personnages de la romancière et la préoccupation de Nagwa Chaban à l'idée de la relation magique entre l'astrologie et le destin des personnages.

Alors, nous distinguons que les deux romanciers explorent l'apport du surréalisme dans l'histoire de la révolution culturelle dans leurs villes. Ces deux romans renoncent le réalisme dans des événements, pour décrire des désirs de changer la réalité. Il est sérieux d'enregistrer que La jument verte et Nawat Alkarm sont apparentés au genre du fantastique. Lord, dans son livre " $L a$ Logique de l'impossible", assure que non seulement le genre fantastique est actuel dans la littérature, mais aussi il produit un commentaire sur le social. Il écrit que la littérature des années 1960 «se caractérise par des æuvres d'auteurs venus d'horizons divers, [...] aux tendances fort variées, allant du merveilleux au fantastique en passant par le réalisme magique et le réalisme étrange, le réalisme en tant que tel et la science-fiction $»^{2}$ Le lecteur admet dans la jument verte et Nawat Elkarm l'intégration d'événements raisonnablement impossibles car les phénomènes

لكا دخلت الشمس برج الاسد فى شهر مسرى حيث وعود الفيضان والخصب...عاد ليث الدين... بثر دمياط ذلك اليوم " I Ibid) p 83 .

(2)-LORD, M-(1995)-La Logique de l'impossible, Québec, Nuit blanche, p. 13. 
Le réalisme magique dans La jument verte de Marcel Aymé et Nawat Alkarm de Nagwa Chaban Dr. Ahmed Moawad Abd-Elhadi

surnaturels sont donc présentés comme s'ils étaient des phénomènes ordinaires. "C'est la résolution antinomique qui fait fondre les deux codes. Le lecteur RM accepte cet amalgame." En effet, pouvons-nous dire que les deux textes posent ainsi en vérité une vision particulière de voir le monde: ils exposent des expériences quotidiennes comme des faits étranges.

Après avoir exposé des étiquettes magiques à travers les traitements religieux comme une sorte du réalisme magique, nous allons discuter un autre type d'images trompeuses; ce sont les rêves, mais il faut remarquer que tout songe n'est pas nécessairement un mensonge. Le rêve est un processus physiologique « qui se déroule pendant le demi-sommeil et qui soit a un caractère de réalité, soit est marqué par un fantastique prodigieux» ${ }^{2}$ Le rêve est une embûche magique d'exposer les désirs et les vœux qui sont difficiles à réaliser, ces rêves sont des aspects du réalisme magique. Dans la jument verte et Nawat Elkarm, les rêves s'attachent à leurs personnages: le cas d'Honoré qui songe à voyager pour travailler dans une usine en ville parce qu'il a besoin d'argent nécessaire pour élever ses enfants:

" Le plus raisonnable serait peut-être de s'en aller travailler en usine, car il n'y avait guère qu'à la ville où sa femme et ses enfants pussent trouver du travail. "Pourquoi pas? Songeait Honoré. Alphonse travaille bien en usine, lui»" 3

Dans cette citation, il y a une étiquette magique qui pousse Honoré à quitter sa terre, son village, son champ, après quarantecinq ans, pour rechercher du travail, car il a besoin d'argent. Donc, le rêve est en relation étroite avec les actes et les pensées de l'homme. "Les peuples qui vivaient à l'état de nature s'en

(1) - SCHEEL, Charles W, op.cit, p. 111.

(2)- Dictionnaire des rêves de A à Z, op. cit, p. 11

(3)- La jument verte, pp. 68,69 -

April 2018 
étaient déjà rendu; c'est pourquoi ils attribuaient au rêve des vertus de seconde vue et de prophétie. Depuis que l'art existe, la littérature, la musique et les arts plastiques sont aussi des moyens d'exprimer les visions de rêves auxquelles l'artiste réfléchit.» ${ }^{1}$

Daisne souligne l'étude entre les éléments de rêve et de magie et la matrice réaliste du texte. C'est ainsi que l'art de Daisne tend à évacuer de plus en plus le fantastique pour exploiter une magie qui incombe presque exclusivement au style. Il cherche "à harmoniser le point de vue de l'enfant avec la sagesse de l'homme mûr." ${ }^{2}$ En dépouillant peu à peu sa prose des événements fantastiques. Marcel Aymé cite un autre rêve qui est considéré comme un pan magique, ce rêve s'attache aussi à Honoré, cet homme malade, qui a songé d'une grande famille: " Honoré aimait encore sa femme à cause des enfants qu'elle lui donnait. Il regardait ses enfants comme des désirs anciens qu'il se réjouissait de retrouver si chaud, avec des yeux vifs et des peaux colorées. ${ }^{3}$

Avec le déroulement du temps, ce rêve se réalise et devient une vérité, cependant, il continue à vivre avec son rêve: "Leurs paroles, leurs chansons, il croyait les dire et les chanter luimême. Ainsi, ses jouissances d'amour étaient sans fin.» ${ }^{4}$ C'est-àdire que le rêve a joué un rôle très important dans sa vie car les rêves " reflétaient l'intérieur de l'homme et permettaient des déductions sur son état psychologique et physique.» ${ }^{5}$

Pouvons-nous envisager la jument verte et Nawat Elkarm dans ce qu'on peut nommer avec Milan Kundera" le grand

(1)- Dictionnaire des rêves de A à Z, op. cit, p. 11

(2)- DUPUIS, Michel "III Flandre et Pays-bas", Le réalisme magique- Roman-Peinture- Cinéma, Jean Weisgerber, p. 94

(3)- La jument verte, p. 89

(4)- Loc.cit, p. 89.

(5)- Dictionnaire des rêves de A à Z, op. cit, p. 12

April 2018 
Le réalisme magique dans La jument verte de Marcel Aymé et Nawat Alkarm de Nagwa Chaban Dr. Ahmed Moawad Abd-Elhadi

contexte ${ }^{\prime l}$ c'est-à-dire qu'il n'y a pas un texte littéraire sans autres ressources. Roussos marque avec conviction la relation indéniable entre le texte et le contexte culturel du roman réaliste magique. De toute évidence, cette relation étroite fait appel à une approche sociocritique. On ne peut pas dire que les deux romans sont des romans merveilleux seulement car le roman merveilleux " est non-thétique, c'est-à-dire qu'il ne pose pas la réalité de ce qu'il représente. $»^{2}$ Mais ce sont deux romans fantastiques aussi parce qu'ils sont: «thétique; il pose la réalité de ce qu'il représente [...] le récit fantastique est issu de la contamination des méthodes de composition des deux types de narration.» ${ }^{3}$ L'écriture chez les deux romanciers dévoile la présence massive du lexique de la société locale soit à Claquebue, soit à Damiette. Les animaux sont sous leur forme physique ou symbolique, ils manifestent un des traits caractéristiques de l'écriture de Marcel Aymé et Nagwa Chaban. Ils font partie intégrante et originel de la culture et de la tradition française ou égyptienne.

Les images animales donnent une puissance essentielle aux aspects magiques dans les deux romans. Les deux romanciers ont donné un nouvel écart à leur langue magique en employant surtout la métaphore et la comparaison. Clles-ci s'attachent à dépasser le sens premier des mots et à leur conférer des connotations. Dans Nawat Elkarm, l'image de la comparaison est fréquente, nous remarquons que Lail, cette vieille fille, imagine l'homme amant comme un monstre. Elle éprouve à son égard une agressivité contre lui qui se manifeste dans les métaphores animales suivantes: Il a apparaît comme un monstre abruti"4, " comme un gorille mâle"5. Ce rêve traduit la haine contre une

(1)- KUNDERA, Milan, op.cit, p. 49.

(2)- BESSIERE, Irène, Le récit fantastique, Paris, Librairie Larousse, (1974), p. 36.

(3)- Ibid, pp. 36-37.

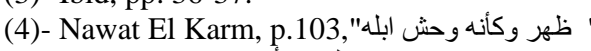

(5)- Ibid, بدا لهاوكأن ذكر غوريلا" p.257 " 
société masculine qui méprise la femme. Ces images animales "métaphorisent l'état psychique du personnage dans le roman."

( $\left.{ }^{1}\right)$ L'image de bête " anomale » exprime la souffrance et la transgression de l'être humain exclu qui est une "problématique humaine "2. Cette image animale montre l'agressivité de cette femme contre un homme esclave cruel. Lail souffre toujours de l'image animale de cet homme.

Autre rêve s'oppose au rêve précèdent en montrant la souffrance de la femme :" à cette nuit, Lail a rêvé qu'elle a soif, et en même temps, elle a un verre d'eau vide, elle cherche quelqu'un pour le lui remplir ce verre......" ${ }^{3}$ Dans ce rêve, nous trouvons que Lail qui voudrait faire l'amour avec un homme convenable, ne le trouve pas. C'est un rêve qui exprime sa volonté refoulée et décrit sa souffrance. Ces images de métaphores et de comparaisons avec des animaux sauvages s'attachent à une caractérisation à la fois morale et physique et se présentent en très grand nombre dans Nawat El Karm. Sinaniya et Lail " sont reléguées aux marges de l'humain, elles sont perçues comme des monstres, mi-bêtes, mi-humains. ${ }^{\prime 4}$ Elles assurent ainsi l'image de l'animalité.

L'image de l'homme cheval et la femme jument se manifeste comme une figure qui réunit deux expressions à deux concepts d'isotopie distincte l'animalité et l'humanité:" Lail a rêvé qu'elle se trouve sur une colline de sable dans une nuit lunée..., elle voit un homme cheval et une femme jument qui dansent et s'embrassent.... ${ }^{\prime 5}$ L'image de ce couple symbolise la

(1) Jean-Michel ADAM et Petit Jean ANDRE, le texte descriptif, poétique historique et linguistique textuelle, Nathan, Paris1989, pp.56-57.

(2 ) Alicia H. PALEO, "La Plume des bêtes: Les animaux dans le roman" in: L'Esprit Créateur, Volume 51, N0 4, 2011, p. 117.

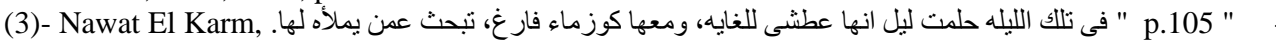

(4) Maria Fernanda ARENTSEN, "Lise Gaboury-Diallo: entre l'esprit cartésien et le réalisme merveilleux", Cahiers franco-canadiens de l'ouest Université de Saint-Boniface, vol. 24, nos 1 et 2, 2012, p. 58.

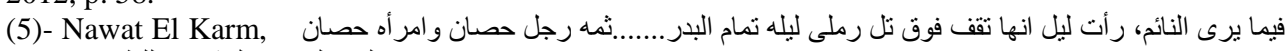

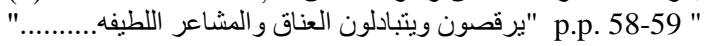


Le réalisme magique dans La jument verte de Marcel Aymé et Nawat Alkarm de Nagwa Chaban Dr. Ahmed Moawad Abd-Elhadi

libération par connaissance spirituelle et la virilité extrême. Lail a besoin d'un homme fort pareil à l'homme cheval. Nagwa Chaban paraît consciente du choix de ce terme. Son utilisation de ce tableau animal illustre bien la nécessité de l'amour. Cette métaphore animale " passe de l'abstrait au concret(...) du non humain à l'humain, (...) de l'inanimé à l'animé, etc." ${ }^{1}$ Alors que l'image du cheval se manifeste au début et à la fin du roman de Marcel Aymé d'une image un peu différente. "Un professeur du collège impérial ...,vint voir la jument verte." ${ }^{2}$ L'image du cheval correspond à la conception de Marcel Aymé qui accentue l'état primitif d'un professeur vis-à-vis de l'existe, du cheval vert et sa réaction irrationnelle. Cette image indique que le cheval vert parle et raconte l'histoire dès le début du roman.

En effet, Marcel Aymé et Nagwa Chaban décrivent des êtres en donnant la forme caractéristique de l'ensemble, il s'agit de préciser des traits animaux couramment esquissés. La métaphore animale est: " le trope qui réalise un transfert sémantique par association d'idées hors contexte" ${ }^{33}$ Bessière pose que le récit fantastique "reflète, sous l'apparent jeu de l'invention pure, les métamorphoses culturelles de la raison et de l'imaginaire $»^{4}$. Mais Todorov considère le fantastique comme un genre en citant que :" Rien ne nous empêche de considérer le fantastique précisément comme un genre toujours évanescent. Une telle catégorie n'aurait d'ailleurs rien d'exceptionnel. ${ }^{15:}$

Un autre rêve qui représente une étiquette magique et fantastique de Hélène Brochard où la femme de Ferdinand Haudouin n'accepte jamais sa vie avec son mari, elle transforme son monde réel par une embuche magique, à songer toujours à la

(1) Daniel BERGEZ et al, Vocabulaire de l'analyse littéraire, Paris, Dunod, 1994 p.135.

(2)-La jument verte, P. 10

(3)-Daniel BERGEZ et al, p.135

(4)-BESSIERE, op.cit, p. 10

(5)- TODOROV, T, op. cit, p. 47.

April 2018 
vie militaire, aux salons des dames d'officiers: "Hélène s'était toujours senti du penchant pour les militaires. (...), elle rêvait au moins une fois par semaine qu'elle était enlevée par un souslieutenant. (...) Elle rêvait aux salons des dames d'officiers où les pianos étaient à queue et où les fauteuils n'avaient point de housses.» ${ }^{l}$ L'auteur présente dans cette citation, une étiquette magique d'une femme qui refuse sa vie avec son mari qui travaille comme un vétérinaire, et en même temps, elle ne peut pas changer sa vie, elle se laisse nager dans le monde du rêve pour qu'elle puisse s'adaptée avec sa vie. Assurément, l'aspect socioculturel prime chez Bessière: "Le récit fantastique utilise des cadres socioculturels et des formes de l'entendement qui définissent les domaines du naturel et du surnaturel $»^{2}$.

La jument verte sert de point de départ d'identifier les traits récurrents du réalisme magique dans la littérature française. Dans ce roman, le lien avec le social est clair: ce qui est mis en lumière par le réalisme magique est représentatif du climat socioculturel. Selon Roussos, le réalisme magique " représente la réalité objective, mais incarne aussi la réalité ressentie" ${ }^{\prime \prime}$ Il cite que certains critiques comme Michel Lord, André Carpentier et Geoff Hancock, renforcent que l'utilisation du surnaturel dans le surréalisme, le fantastique et le merveilleux "correspond au besoin de dévoiler un monde où rien n'est jamais fixe et dans lequel prospèrent des sortes aux prises avec le réalisme.» ${ }^{4}$ Citons à l'appui cette embûche magique du maire de Claquebue, Philibert Messelon qui a 72 ans, en répondant aux questions suivantes:-Comment ça fait quand on est mort? Et Qu'est-ce que tu pensais d'être mort?

"Le vieux répondit d'une voix fluette: -Je me suis trouvé en paradis vers les huit heures du matin et

(1)- La jument verte. p. 104.

(2)- Ibid, p. 11.

(3)- Roussos, K., op. cit, p.34. -

(4) - Ibid, p.34. 
Le réalisme magique dans La jument verte de Marcel Aymé et Nawat Alkarm de Nagwa Chaban Dr. Ahmed Moawad Abd-Elhadi

rajeuni de cinquante ans, comme vous pensez. C'est un endroit plaisant où il y a de la fraîcheur et de la distraction. Le bon Dieu s'est montré bien honnête, et pas fier comme il y en a qui voudraient le faire croire. "Puisque vous arrivez, Philibert, qu'il m'a dit, je m'en vais faire un tour de paradis avec vous.» ${ }^{1}$

A vrai dire, Marcel Aymé présente une image formidable en modifiant de cette vie à la vie dernière, non seulement, il parle de cette vie, mais aussi, il raconte l'histoire de Philibert Messelon dans cette vie. Le narrateur emmène le lecteur avec lui dans un voyage céleste au paradis en limitant le temps, l'espace et l'âge; le paradis qui signifie pour lui" une récompense extraterrestre pour compenser l'ingratitude de son entourage.» ${ }^{2}$ à huit heures du matin, c'est le meilleur temps de la journée, l'endroit plaisant avec le vin de paradis et il ne faut pas oublier qu'il a cinquante ans, âge de sagesse. Remarquons qu'il montre ses besoins. Ajoutons aussi qu'il montre une embûche magique merveilleuse en citant qu'il a rencontré le bon Dieu qui lui a proposé de faire un tour de paradis avec lui. C'est ainsi que l'auteur figure un réalisme magique en utilisant une étiquette magique pour exprimer et approcher aux lecteurs une image métaphysique. Bien que ce rêve soit extraordinaire, il est présenté d'une manière suprême. Enfin, il ne faut pas dire que tous les rêves sont agréables, "les rêves ne procurent pas tous du plaisir et n'apportent pas tous la solution des problèmes qu'ils posent. Les rêves peuvent aussi être inconfortables, aussi inconfortables que la vérité.» ${ }^{3}$

(1)- La jument verte, pp.184, 185

(2)- Dictionnaire des rềves de A à Z, op. cit, p. 211

(3)- Ibid, p. 14

April 2018 
$\mathrm{Au}$ terme de cette modeste recherche, nous pouvons dire que les traitements des embûches magiques dans la jument verte et Nawat Elkarm nous permettrent de captiver un regard original sur le réalisme magique en tant que genre de la littérature contemporaine. Aussi, le réalisme magique de Roh met le surprenant au sein de la réalité. Indiquant que le réalisme magique est un recommencement de la réalité, "un retour effectué après un détour expressionniste qui grave décisivement la notion de l'impossible dans cette nouvelle vision réaliste"l. C'est-à-dire que le travail de Marcel Aymé et Nawat Elkarm subit encore "l'influence du fantastique, du post-expressionnisme et du surréalisme visant un réalisme socialiste imprégné de l'histoire américaine" ${ }^{\prime 2}$ Nous supposons que, malgré l'espace différent, les personnages et les événements différents, l'évasion du réel amer crée le réaliste magique dans les deux romans afin que les personnages puissent résoudre les problèmes physiques et moraux. La souffrance "conçue comme partie de la condition humaine" $^{3}$ est une image apparente dans tous les siècles. L'écriture est considérée comme une thérapie contre elle. L'artiste est, seul, apte à la dessiner en se servant de sa plume. Marcel Aymé et Nagwa Chaban ont mis cette souffrance dans le cadre du réalisme magique. Ajoutons que la jument verte et Nawat Elkarm ont un charme qui fait intervenir dans un cadre réaliste des événements magiques, ces événements surnaturels sont généralement acceptés par les personnages, et sont empruntés aux mythes et aux croyances d'une culture spécifique. Tandis que dans le roman fantastique, le cadre est réaliste, il y a des éléments étrangers, en général, il recherche à refuser le possible du surnaturel.

(1)- ROH, F, op.cit. pp. 17,18

(2)- Roussos, K, op.cit, p.29.

(3) Jacques G. RUELLAND, "Que devrait être la paix ? ", in La libre pensée québécoise, Montréal, no 10 (1er semestre 1989), p.21. 
Le réalisme magique dans La jument verte de Marcel Aymé et Nawat Alkarm de Nagwa Chaban Dr. Ahmed Moawad Abd-Elhadi

Mentionnons que l'irréel de Marcel Aymé gagne à être détourné à sa campagne habituel parce que la critique de l'œuvre est absolument figée, "le fait de considérer les æuvres littéraires à l'échelle internationale conduit à découvrir d'autres principes de contigüité ou de différenciation, qui permettent de rapprocher ce qu'on sépare d'ordinaire et de séparer quelquefois ce qu'on a coutume de rassembler." Généralement, l'auteur cherche à tisser des liens étroits entre des courants habituellement opposés tels que le naturalisme, le merveilleux et le fantastique afin de " peindre une réalité transfigurée par l'imaginaire et dans laquelle le rationalisme est rejeté.» ${ }^{2}$ Finalement, on est d'accord avec l'analyse de Chanady qui supporte que le fantastique et le réalisme magique soient des modes narratifs, bien différents quoique très proches.

Marcel Aymé offre une étude sociale à travers la jument verte, avec un vocabulaire précis pour chaque type humain. Son langage est d'ailleurs "un des plus riches de la littérature contemporaine. " 3 Il est resté très attaché à sa région d'origine, la Franche-Comté, à laquelle il a fait une place de choix dans son roman. Il y a une peinture complexe du milieu qu'il observe et une vérité psychologique sous chaque personnage. A vrai dire, La jument verte est un roman du réaliste magique car Marcel Aymé met en évidence une pensée spéciale depuis son titre qui annonce un réaliste magique, aussi, on remarque que la manière de présenter l'histoire est dénormale et hors du commun de regarder le monde.

Bien que Nagwa Chaban ne mette pas en évidence une pensée spéciale depuis son titre, mais nous affirmons, après une

(1)- Ibid, P. 244

(2)- «Le réalisme magique latino-américain » [archive], sur Scyfy (consulté le 6 mai 2014)

(3)-La Comédie humaine de Marcel Aymé, La Manufacture, Lyon, 198, p. 259-265(ISBN 978-2-03508302-9)

April 2018 
lecture sérieuse, que Nawat Elkarm est un roman réaliste magique. Elle présente une étude sociale en utilisant un lexique précis pour chaque type humain. Il y a un portrait précieux de la société qu'elle observe et une vérité psychologique sous chaque individu. Pour Nagwa Chaban, l'embûche magique est un instrument de combat contre le pouvoir caché derrière la tradition: l'oppression, les contraintes sociales contre le mariage. L'utilisation fréquente des mots clefs d'astrologie figure la focalisation de Nagwa Chaban de la condition des misérables à Damiette. Elle a emprunté des expressions magiques, simples et libres de la gaieté du langage populaire. Nagwa Chaban avait tenu la cadence qu'elle donne à son roman dans les premières pages jusqu'au bout. Nagwa Chaban demeure son roman bien agréable à lire, souvent drôle, où elle ne se prend pas au sérieux et exprime les blocages du désir de ses personnages à travers le regard d'une fille qui était le témoin de l'intérieur de tout ce petit théâtre honteux du quotidien. La campagne et les mœurs rurales sont évoquées avec une gaillarde bonne humeur, simplicité et naturel. Les changements de focalisation sont prévenus par "l'héroïne Lail" qui nous rapporte des faits qu'elle seule a pu voir ! C'est dans ces passages que l'on trouve un bon nombre d'épisodes érotiques.

Chaque romancier nous présente son langage magique mais selon sa perspective. La manière d'adopter la langue magique chez les deux romanciers met en valeur la culture et la tradition de deux univers différents en montrant l'idéologie de deux romanciers à propos de l'homme et la femme dans la société. Dans Nawata Alkarm comme dans la jument verte, les termes magiques sont des actes de création qui résultent de la culture locale égyptienne ou française. Cela justifie l'existence des concepts surnaturels à travers les hommes de la religion chrétienne et musulmane ou des magiciennes qui entrent en ligne comme complice dans la magie. L'occurrence des métaphores 
Le réalisme magique dans La jument verte de Marcel Aymé et Nawat Alkarm de Nagwa Chaban Dr. Ahmed Moawad Abd-Elhadi

animales dans les deux romans reflètent une réalité culturelle. La métaphore est née de la perception, de l'imagination et des rêves.

Nous avons essayé de dire qu'il y a une ressemblance qui compose modèle remarquable du réalisme magique chez Marcel Aymé et Nagwa Chaban qui exposent à travers leurs ouvrages des images formidables et magiques dans un style très élaboré. Ils analysent avec esprit les travers de l'homme et de la société. Leur vision peut être noire. Ils peignent les structures sociales de façon très réaliste, tout en accordant une place importante au fantastique. Ils ont l'art de mettre en scène toutes les classes sociales avec le langage qui leur est propre. 


\section{Bibliographie}

\section{Corpus de l'étude:}

AYME, Marcel, La jument verte, Paris, Gallimard, (1933). ـ نجوى شعبان، نوة الكرم، القاهرة ، الهيئة الهصرية العامة للكتاب، 2006

\section{Ouvrages de critique:}

(1) ADAM, Jean-Michel et ANDRE, Petit Jean, Le texte descriptif, poétique historique et linguistique textuelle, Nathan, Paris1989, pp.56-57.

(2) - ARENTSEN, Maria Fernanda "Lise Gaboury-Diallo: entre l'esprit cartésien et le réalisme merveilleux", Cahiers franco-canadiens de l'ouest Université de Saint-Boniface, vol. 24, nos 1 et 2, 2012.

(3) -BERGEZ, Daniel et al, Vocabulaire de l'analyse littéraire, Paris, Dunod, 1994.

(4) -BESSIERE, Irène, Le récit fantastique, Paris, Librairie Larousse, (1974)

(5) -CASANOUVA, Pascale, La République mondiale des lettres, Paris, Seuil, (1999)

(6) - CHANAD, Amaryll, Entre inclusion et exclusion- Paris, Honoré champion. (1999)

(7) -DUPUI, M et MINGELGRUN, A, "Pour une poétique du réalisme magique" Le réalisme magique- RomanPeinture-Cinéma, Jean Weisgerber, (1987)

(8) - FUENTES, Carlos, le sourire d'Erasme: Epopée, utopie et mythe dans le roman hispano-américain, Paris, Gallimard(Le Messager) (1992)

(9) - GENETTE, G., Nouveau discours du récit, Seuil, Poétique, (1983)

(10) - HELLENS, Franz, Le fantastique réel, Bruxelles, Sodi, (1967)

(11) - KUNDER, Milan, Le Rideau- Paris, Gallimard, (2005) 
Le réalisme magique dans La jument verte de Marcel Aymé et Nawat Alkarm de Nagwa Chaban Dr. Ahmed Moawad Abd-Elhadi

(12) - LORD, M, La Logique de l'impossible, Québec, Nuit blanche, (1995)

-PAGEAUX, Daniel-Henri, 'Préface", Charles W. Scheel" Réalisme magique et réalisme merveilleux: des théories aux poétique, Paris, L'Harmattan, (2005)

(14) -PALEO, Alicia H. "La Plume des bêtes: Les animaux dans le roman" in: L'Esprit Créateur, Volume 51, N0 4, 2011.

(15) - ROH ,Franz, Nach-expressionismus (Magischer Realismus: Probleme der neuesten europäischen Malerei, Leipzig, Klinkhardt \& Biermann, (1925) (traduction française avec présentation et notes par Jean Reubrez Postexpressionnisme Réalisme magique Problèmes de la peinture européenne la plus récente, Dijon Les presses du réel 2013 )

(16) - ROUSSOS, Katherine, Décoloniser l'imaginaire:leréalisme magique chez Maryse Condé, Sylvie Germain et Marie Ndiaye, Paris, L'Harmattan, (2007)

(17) -SCHEEL, Charles $\mathrm{W}$, Réalisme magique et réalisme merveilleux- Des théories aux poétiques, Paris, L'Harmattan, (2005)

(18) -TODOROV, Tzvetan, Introduction à la littérature poétique- Paris, Ed. du Seuil, (Coll. «Points/Essais), (1976)

-TROUSSON,Raymond, "Du fantastique et du merveilleux au réalisme Peinture, (1987)

(20) - WEISGERBER, Jean, (dir), Le Réalisme magique,:roman, peinture et cinéma, "La locution et le concept", Bruxelles, L'âge d'Homme, (1987) 
Thèses:

(21) -GIGUERE, Marielle, Julien Gracq et le réalisme magique, Mémoire soumis à l'Université McGill en vue de l'obtention du grade de $\mathrm{M}$. A. en langue et littérature françaises, Monteral, (2007)

\section{Dictionnaires:}

(22)-KURTH, Hanns, Dictionnaire des rêves de A à Z, France Loisirs, Paris, boulevard de Grenelle, (1977)

(23)- SCHMITT,Michel P. dans : Dictionnaire des littératures de langue française, Bordas, vol. I, 1984, p. 109 (ISBN 978-2-04015333-5 -

(24)- Dictionnaire historique, thématique et technique des littératures, (1985)- vol. I. Larousse, (ISBN 978-2-03-5083012).

\section{Internet:}

(25)-«Le réalisme magique latino-américain » [archive], sur Scyfy (consulté le 6 mai 2014)

(26)-La Comédie humaine de Marcel Aymé, La Manufacture, Lyon, 1985, p. 259-265 (ISBN 978-2-03-508302-9). 
Le réalisme magique dans La jument verte de Marcel Aymé et Nawat Alkarm de Nagwa Chaban Dr. Ahmed Moawad Abd-Elhadi

\section{Résumé Le réalisme magique dans La jument verte de Marcel Aymé}

\section{et Nawat Alkarm de Nagwa Chaban}

La production littéraire de Marcel Aymé et Nagwa Chaban est attirée par l'inclusion de l'extraordinaire dans la fiction, c'est parce qu'ils ont reçu une culture traditionnelle dans laquelle les croyances religieuses et les fantasmes sont caractéristiques d'une situation sociale. Notre réflexion porte plus précisément sur la notion du réalisme magique dans la littérature et à travers les deux romans. Dans cette recherche, nous essayerons d'appliquer les théories qui ont rapport au réalisme magique. L'auteur de la Jument verte est un écrivain français, né et mort à Paris. L'auteur de Nawata Alkarm est une romancière égyptienne. Les événements de son roman se passent à Damiette, la terre natale de la romancière au début du $\mathrm{XVI}^{\mathrm{e}}$ siècle. Les deux histoires sont essentiellement des études de la morale sexuelle et des comportements des habitants de deux petits villages français et égyptien. C'est-ainsi que Marcel Aymé et Nagwa Chaban exposent à travers leurs ouvrages une image magnifique pleine d'aspects fantastiques, merveilleux, religieux, rêveurs et magiques dans un style très élaboré. Mots-clefs: embûcheréalisme magique - merveilleux- religion- étiqueté- rêve. 
